Published in final edited form as:

Drug Alcohol Depend. 2012 November 1; 126(1-2): 7-12. doi:10.1016/j.drugalcdep.2012.03.010.

\title{
Increases in the availability of prescribed opioids in a Canadian setting
}

\author{
B. Nosyk ${ }^{1}$, B.D.L. Marshall ${ }^{1,2}$, B. Fischer ${ }^{3,4}$, J.S.G. Montaner ${ }^{1,5}$, E. Wood ${ }^{1,5}$, and T. Kerr ${ }^{1,5}$ \\ ${ }^{1}$ British Columbia Centre for Excellence in HIV/AIDS, St. Paul's Hospital, 608-1081 Burrard \\ Street, Vancouver, BC, CANADA, V6Z 1 Y6 \\ ${ }^{2}$ Department of Epidemiology, Columbia University Mailman School of Public Health, $722 \mathrm{~W} 168^{\text {th }}$ \\ Street, New York, NY, USA, 10032-3727 \\ ${ }^{3}$ Faculty of Health Sciences, Simon Fraser University, 8888 University Drive, Burnaby, British \\ Columbia, Canada, V5A 1 S6 \\ ${ }^{4}$ Centre for Addictions and Mental Health, 33 Russell St., Toronto, Ontario, Canada M5S 2S1 \\ ${ }^{5}$ Department of Medicine, University of British Columbia, St. Paul's Hospital, 608-1081 Burrard \\ Street, Vancouver, BC, CANADA, V6Z 1 Y6
}

\begin{abstract}
Background-The nonmedical use of prescribed opioids (POs) has increased across North America over the past decade. Our objective was to identify changes in the availability of POs and other illicit drugs among drug users in a Canadian setting.
\end{abstract}

\begin{abstract}
Methods-Information on the availability of illicit drugs was collected in standardized interviews from a large observational research program involving illicit drug users in Vancouver, British Columbia from 2006-2010. The primary outcome was the perceived availability of a set of six POs (aspirin/oxycodone, hydromorphone, oxycodone, morphine, acetaminophen/codeine and methadone) among individuals reporting ever using POs. Availability was measured in three levels: not available, delayed availability (available $\geq 10 \mathrm{~min}$.), and immediate availability (available <10min.). Multivariate ordinal logistic regression models were executed to estimate the trend in PO availability, controlling for individual characteristics hypothesized to influence availability.
\end{abstract}

Results-1,871 individuals were followed during the study period (2006-2010), including $583(31.2 \%)$ women. The availability of POs increased over time, regardless of changes in the characteristics of cohort entrants. These increases were observed while the availability of traditional drugs of abuse (e.g., heroin and cocaine) remained constant. The adjusted odds of

(C) 2012 Elsevier Ireland Ltd. All rights reserved.

Send correspondence to: Bohdan Nosyk, B.C. Centre for Excellence in HIV/AIDS, University of British Columbia, St. Paul's Hospital, 613-1081 Burrard Street, Vancouver, B.C., V6Z 1Y6, Canada, Tel: (604) 806-8649, Fax: (604) 806-9044, bnosyk@cfenet.ubc.ca.

Contributors: Authors BN, EW and TK and BF designed the study protocol. BN wrote the first draft of the manuscript. BN undertook the statistical analysis with significant scientific input from BDLM and TK. TK, EW and JSGM were responsible for data collection. All authors contributed to and have approved the final manuscript.

Conflict of interest: All authors declare that they have no conflicts of interest.

Publisher's Disclaimer: This is a PDF file of an unedited manuscript that has been accepted for publication. As a service to our customers we are providing this early version of the manuscript. The manuscript will undergo copyediting, typesetting, and review of the resulting proof before it is published in its final citable form. Please note that during the production process errors may be discovered which could affect the content, and all legal disclaimers that apply to the journal pertain. 
delayed availability vs. unavailability were between $34 \%$ (hydromorphone) and $71 \%$ (acetaminophen/codeine) greater in each calendar year.

Discussion-The availability of POs among drug users in a Canadian setting increased markedly over a relatively short timeframe, despite persistent and high availability of heroin and cocaine. Further study is required to determine the context of use of POs, associated harms, as well as policy responses to increasing availability.

\section{Keywords}

Prescribed opioids; availability; oxycodone; hydromorphone; methadone; illicit drugs

\subsection{Introduction}

Whereas the 1980s and 1990s bore witness to epidemics of crack cocaine and heroin use in many inner city areas, the nonmedical use of prescribed opioids (POs) is emerging as an increasing concern. Rising rates of use have been documented in the US, most notably among youth and young adults. Among high school seniors, Hydrocodone abuse was second only to marijuana abuse (Volkow and McLellan, 2011). A 7-fold increase in drug treatment admissions involving opioids other than heroin was observed between 1998 and 2009. (SAMHSA TEDS Database, 2011). Emergency department visits involving non-medical use of prescription drugs increased to a greater extent than other illicit drugs, with Oxycodone $(175,949$ in 2009; a $242.2 \%$ increase from 2004) and Hydrocodone (104,490 in 2009; a $124.5 \%$ increase from 2004) among the highest increases. Finally prescribed opioid-related overdose deaths increased from 2000 in 1999 to 14,800 in 2008. (Centers for Disease Control and Prevention, 2011). Opioid overdose is now the second leading cause of unintentional death in the United States, second only to motor vehicle accidents (National Centre for Injury Prevention and Control, 2010), which prompted the Centers for Disease Control and Prevention to label PO overdose as a national epidemic (Centers for Disease Control and Prevention, 2011).

There is accumulating evidence of similar trends in use in Canadian settings. An early study conducted in Vancouver identified a range of prescription medications available for illicit sale, including POs such as acetaminophen/codeine, aspirin/oxycodone, meperidine, hydromorphone, morphine, and Anileridine (no longer manufactured in North America; Sajan, 1998). In a 2004 report on a cohort of methadone maintenance treatment (MMT) patients in Ontario, Brands et al. (2004) reported that 83\% of all patients had been using POs, with or without heroin, upon admission. The OPICAN study, conducted in five Canadian cities, revealed that nonmedical use of POs was far more prevalent than the use of heroin in every setting except Vancouver and Montreal (Fischer et al., 2005). From 2002 to 2005 , a relative increase of $24 \%$ was observed in the proportion of the street drug using population who used non-medical POs only (Popova et al., 2009). Recent studies have described increases in the amounts of opioids prescribed across Canada (Fischer et al., 2011), increasing opioid utilization among recipients of social assistance in Ontario (Gomes, 2011a) and street users in Montreal (Bruneau et al., 2012; Roy et al., 2011), a strong independent relationship between PO dose and opioid-related mortality (Gomes, 2011b), high variation in opioid prescribing among Ontario physicians (Dhalla, 2011) and high rates of non-methadone opioid use among clients in methadone maintenance treatment in Ontario (Kurdyak et al., 2011).

Despite this rapidly growing literature documenting problematic PO use in North America, few studies have endeavored to ascertain temporal trends in street-level availability of POs and other illicit drugs (e.g., cocaine and heroin). Our objective, therefore, was to examine 
the availability of prescribed opioids and other illicit drugs among street users in Vancouver, British Columbia between 2006 and 2010.

\subsection{Methods}

\subsection{Study design}

Data for this analysis were derived from the baseline assessments of a series of ongoing open prospective cohort studies involving illicit drug users, including the At-Risk Youth Study (ARYS), the AIDS Care Cohort to evaluate Exposure to Survival Services (ACCESS), and the Vancouver Injection Drug Users Study (VIDUS). The VIDUS study began enrollment in May 1996 and recruits individuals through word of mouth, street outreach, and referrals. Recently, the original VIDUS cohort was divided into two separate studies: VIDUS now follows HIV-negative participants and its sister study ACCESS follows HIV-positive drug users based in the Greater Vancouver area (Strathdee et al., 1998; Wood et al., 2009). The At-Risk Youth Study began in late 2005 and is made up of street-involved youth who report use of drugs other than or in addition to cannabis and are aged 14 to 26 (Wood et al., 2006).

Sampling and follow-up methodologies have been described in detail previously (Strathdee et al., 1997; Tyndall et al., 2003; Wood et al., 2006). Specific eligibility criteria were specified in other articles; however, general eligibility across all three cohorts required age of at least 14 years, Greater Vancouver region residence, and the provision of informed consent. At baseline, participants complete an interviewer-administered questionnaire that elicits information pertaining to sociodemographic characteristics, drug use, treatment utilization, and HIV risk behaviours. Nurses also assessed participants for various health conditions, and obtain blood specimens for HIV and Hepatitis C Virus (HCV) serology, and HIV disease monitoring (e.g., CD4 counts, HIV-1 RNA) where appropriate. Participants receive \$20 CAD for each visit. While combining data from studies with different inclusion criteria may present some challenges, we note that all studies rely on harmonized recruitment and data collection tools. These studies have been approved by the University of British Columbia/Providence Health Care Research Ethics Board.

\subsection{Participants and measures}

Questions assessing the availability of illicit drugs were first added to the baseline study instrument for the ARYS, ACCESS and VIDUS cohort studies in late 2005. All participants who completed the baseline interview after this date were eligible for inclusion. The study period was defined as the five-year interval ending in December 31st, 2010. The availability of a set of 12 substances (crack cocaine, heroin, powder cocaine, crystal methamphetamine, marijuana, aspirin/oxycodone, hydromorphone, oxycodone, morphine, acetaminophen/ codeine and methadone), acquired illicitly, were assessed at five levels: (1) score within 10 minutes; (2) score within 90 minutes; (3) score within a day; (4) score in more than a day; (5) could not score this drug.

We assessed changes in the availability of these drugs over the study period, with availability being an aspect of supply, rather than demand for the substances in question. Given that individual characteristics could influence access to a given illicit drug, and these factors may have changed among cohort entrants over the study period, we controlled for these factors by estimating the independent effect of calendar year on the availability of illicit drugs among cohort entrants. Further, respondents were asked to provide assessments of availability regardless of whether they had ever used the substance. We included assessments on availability from individuals who reported having used the substance in question. 
We hypothesized that a number of factors were potentially associated with availability. Aside from age and gender, we hypothesized unstable housing (defined as living in a single occupancy room hotel, a treatment or recovery house, jail, shelter or hostel, or having no fixed address for the last six months), daily use of POs, drug dealing involvement, sex work involvement, and geographic proximity to Vancouver's Downtown Eastside (DTES) would each influence how quickly an individual may access illicit drugs. Housing instability may be indicative of greater mobility and therefore irregular contacts with suppliers, while daily use and dealing status are clearly indicative of stronger contact with a given drug supply chain. Involvement in sex work, potentially in exchange for illicit drugs, may also increase the ease of availability of illicit drugs. Vancouver's DTES is the most impoverished neighborhood in Canada, and home to a high concentration of illicit drug use (Wood and Kerr, 2006), and therefore proximity to this area may predict easier access to drugs. For the latter, we considered several related variables, including current DTES residence, any DTES residence in the past 6 months, regular visits to the DTES and indication of purchasing illicit drugs in the DTES, and selected the covariate that provided the best model fit, using Akaike's and Bayes' information criteria, and the largest effect size. Unless otherwise indicated, variables refer to behaviours or activities in the past six months from the date of the baseline interview. In addition, we included cohort indicators, given that the assessments were drawn from three separate cohort studies with different aims and target populations.

\subsection{Statistical Analysis}

As a first step, we plotted univariate trends in the availability of each drug assessed in baseline data from participants entering the cohorts in different years. We then constructed ordinal logistic regression models to determine the odds of delayed and immediate availability, controlling for other factors. The proportional odds assumption was tested using the Score test. Given low levels of responses for availability in $<90$ minutes, $<1$ day and $>1$ day, we combined these categories, thus providing us with a three-level, ordered outcome variable: not available; delayed availability (available in $\geq 10$ minutes) and immediate availability (available in $<10$ minutes). We proceed with this terminology from this point onward. Regression models for the availability of each substance were constructed with manual stepwise elimination. For dichotomous variables, adjusted odds ratios are interpreted as the increase/decrease in the odds of the higher availability category for a 1-unit increase in the covariate (in comparison to the stated reference group). All analyses were executed using SAS version 9.2 .

\subsection{Results}

\subsection{Summary statistics}

Summary statistics on the covariates included in the analysis are provided in Table 1. A total of 1871 individuals were recruited into the ARYS [N=712 (38.1\%)], ACCESS [N = 536 $(28.7 \%)$ and VIDUS [ $\mathrm{N}=623(33.3 \%)$ ] cohorts during the study period; $31 \%$ were female and $37 \%$ were 25 or under (median age: 33.7 ; interquartile range: $22.7,44.3$ ). The majority of individuals reported living in unstable housing (52\%), 37.8\% reported dealing drugs and $8.3 \%$ reported being engaged in sex work in the past 6 months. Past use of each of the major classes of substances was high; 66.3\% the cohort reported ever having used POs in the past. Finally, in each calendar year, between 123 (6.6\%) in 2010 and 643 (34.4\% in 2006 individuals entered the study and completed baseline questionnaire packages.

\subsection{Univariate trends in availability}

Trends in the immediate availability of the illicit drugs assessed are plotted in Figure 1. While the immediate availability of heroin, crack cocaine, powder cocaine, crystal methamphetamine and marijuana were high and remained constant throughout the study 
period (Figure 1), the immediate availability of POs all increased between 2006 and 2010. Increases in the immediate availability of aspirin/oxycodone and hydromorphone were the most pronounced, increasing by $26.7 \%$ and $19.8 \%$, respectively, for these substances during the study period. Immediate availability of aspirin/oxycodone increased from $11.2 \%$ in 2006 to $37.9 \%$ in 2010; hydromorphone increased from $21.6 \%$ to $41.4 \%$, and acetaminophen/ codeine from $17.2 \%$ to $39.7 \%$. During this same period, the proportions of individuals reporting that the set of POs were not available dropped 10-20\%, most notably in 2007 (Figure 2).

\subsection{Multivariate analysis}

Results of the multivariate analysis are provided in Table 2. As the score test indicated odds ratios were not proportional between the levels of availability, we fitted separate logistic regression models assessing the odds of delayed availability vs. unavailable and immediate availability vs. delayed availability. Controlling for other covariates, the odds of delayed availability was between $34.0 \%$ (hydromorphone) and $71.0 \%$ (acetaminophen/codeine) greater in each given calendar year, compared to being unavailable. Drug dealing status was significantly associated with ease of availability for each of the substances assessed, while daily use was associated with higher odds of immediate availability (compared to delayed availability) of all but acetaminophen/codeine and methadone. Morphine, oxycodone and acetaminophen/codeine were generally more immediately available to older individuals, particularly those over 45 , while female gender, unstable housing and engagement in sex work and buying drugs in the DTES were not associated with availability when adjusting for other factors. Similarly constructed regression models were estimated for each of the other substances presented in Figure 1 - no significant trends in changes in availability were found (data not shown).

\subsection{Discussion}

Among illicit drug users in Vancouver, Canada, the immediate availability of POs increased significantly from 2006 to 2010 . These increases persisted after adjustment for changes in the characteristics of individuals entering the cohorts under study. Our finding suggesting increased presence of POs within illicit drug markets is consistent with a diverse set of studies undertaken in North America indicating rising PO use (Volkow and McLellan, 2011; Fischer et al., 2005; Popova et al., 2009; Rosenblum, 2011). However, to our knowledge, this is the first study to document increased availability of POs alongside high and stable availability of other more traditional drugs of abuse (including heroin).

\subsection{Insights into the market for illicit drugs}

Like any other market for consumer products, the market for illicit drugs has proliferated with a greater variety of options available to buyers. One noteworthy finding is the uninterrupted ease of availability of the more traditional illicit drugs found in Vancouver. Illicit drugs have remained readily available to drug users while policing intensity and expenditures on enforcement have continued to rise (BC Stats, 2011), and the period under study has featured several highly-publicized violent conflicts among rival drug gangs, reportedly due to breaks in upstream supply chains (CTV News Report, 2009). The durability of the supply and use of illicit drugs in Vancouver was previously demonstrated during a police crackdown (Wood et al., 2004) and following a large seizure of heroin in the port of Vancouver (Wood et al., 2003). However, in 2001, the effects of an alleged heroin drought was observed in Vancouver and in other settings served by producers within Golden Triangle region, and within Vancouver the drought was associated with short-term decreases in three independent markers of heroin use (Wood et al., 2006). The reasons for this drought have been debated: several commentators believe these changes in heroin use to be a result 
of upstream interruptions in production (Wood et al., 2006). Although high levels of heroin use were quickly restored (Marshall et al., 2011), the increases in PO availability alongside persistent availability of heroin could reflect fluctuating purity of heroin and corresponding increases in demand for POs.

The supply of traditional drugs of abuse is understood to be maintained through adjustments in drug purity - interruptions in the supply chain result in lower-purity product distributed at the retail level (Caulkins, 2006). POs, however, represent an interesting contrast, as they are manufactured by pharmaceutical companies, prescribed by physicians, dispensed from local pharmacies, and have a composition that cannot be easily altered. These characteristics suggest the supply of POs may be more effectively controlled, particularly if the finding that PO users obtain the drugs directly or indirectly through regular pharmacy dispensation (Volkow et al., 2009), is universally true. However, this claim remains to be verified in settings involving long-term or polysubstance users in Canada and elsewhere, and ongoing reports of pharmacy break-ins, prompting policy response in British Columbia (BC College of Pharmacists, 2011) suggest that regulatory changes at the prescribing and dispensation level may not fully address this problem.

\subsection{Implications for medical care}

In light of the presumed supply of non-medically used POs through the medical system, a number of recommendations to control the diversion of POs have been proposed, including: enhanced clinical teaching and training practices; standardized screening procedures and best practice guidelines for managing patients with chronic non-cancer pain; indications for when and how long to prescribe opioid analgesics; indications for when short vs. long acting opioids should be prescribed; appropriate use of urine screening procedures to manage risk of diversion, abuse and addiction; the use of patient contracts; state prescription drug monitoring programs to reduce doctor shopping; as well as continuation and discontinuation criteria (Chou et al., 2009). These types of considerations by Canadian provincial Colleges of Physicians are clearly in need. Response to these matters has also come from drug companies; the US Food and Drug Administration recently approved a new formulation of oxycodone designed to discourage misuse and abuse (Food and Drug Administration, 2010).

Our results indicating the increasing ease of availability of diverted methadone is somewhat concerning, as the potential backlash from this finding may trigger a policy response that would affect availability to those who require it. While each of the other opioids covered in this analysis are prescribed for the management of pain, methadone has been effectively used to treat opioid dependence for over 40 years (Kreek et al., 2010). While it was not possible to assess whether methadone was diverted from prescriptions with indications for pain or opioid dependence, evidence of methadone diversion can compromise both the availability of methadone treatment, as well as the quality of care provided to clients engaged in substitution treatment. Policies on take-home doses of methadone treatment in BC (currently not recommended beyond 7 days) were formulated primarily to minimize the public health risks related to methadone diversion (Ruel and Hickey, 1993). However, takehome doses may allow stabilized patients greater freedom in attending to family life, employment or other productive endeavors. In British Columbia, take-home doses are more often prescribed in rural areas (Nosyk et al., 2010), and may be a necessary component in the reintegration of clients into society, as co-payments are required once an individual gains employment. Studies conducted in the United States have demonstrated success with long take-home doses (up to 1 month) in stabilized, responsive patients (Salsitz et al., 2000; Harris et al., 2006). Evidence on the public safety issues regarding take-home doses should be balanced against evidence that supports its effectiveness. 


\subsection{Contexts of street use of prescribed opioids}

While we've described and discussed the increasing supply of prescribed opioids, the demand, and context of use of these substances requires closer consideration. Davis and Johnson (2008) provided a more nuanced perspective on the matter in an ethnographic analysis of 586 street drug users in New York City. This study revealed that subjects in this study were classified according to whether they diverted POs or used POs to relieve pain or withdrawal rather than for euphoria. PO diversion was associated with frequency of PO use, whether POs were obtained from doctors/pharmacies or from drug dealers and family members. Policy and programmatic responses should acknowledge that POs may be used as a primary drug of abuse, as a temporary solution to opioid withdrawal, or as genuine pain relief. Greater insight into the contexts of street use in Vancouver and elsewhere in Canada are thus clearly needed to inform policy and programmatic responses.

\subsection{Limitations}

Our analysis is not without limitations. Primarily, the extent to which the results are generalizable to the drug using population in British Columbia is uncertain, as the cohorts under study were not explicitly sampled randomly from the population of drug users in Vancouver, BC. Further, while we've attempted to control for the potentially changing mix of participants entering the cohorts during the study period, given the observational nature of this study, there is always a possibility that there may be residual confounding that may either increase, or decrease, the true magnitude in change of availability of each of the drugs assessed. The consistency of our findings with other studies of diverse designs and populations under study however support the direction of our findings. As well, we were unable to assess fluctuations in drug purity, which may in part explain the trends observed herein. Future studies should seek to assess a range of questions regarding the determinants of the demand and supply of POs, including whether changing heroin purity affects the availability and use of POs.

The availability of POs among the study population increased markedly over a relatively short timeframe. This increase occurred despite the high and stable availability of other, more traditionally used illicit drugs, including heroin. Further study is required to determine the context of use of POs and, subsequently, appropriate policy and programmatic responses.

\section{Acknowledgments}

The authors thank the study participants for their contribution to the research, as well as current and past investigators and staff. We would like to thank Deborah Graham, Peter Vann, Caitlin Johnston, Steve Kain, and Calvin Lai for their research and administrative assistance.

Role of funding source: Funding for this study was provided by National Institutes of Health (NIH) grants R01DA011591 and R01-DADA031727 and a Canadian Institutes of Health Research (CIHR) grant RAA-79918. Thomas Kerr is supported by a Scholar Award from the Michael Smith Foundation for Health Research (MSFHR) and an Investigator Award from the CIHR. Bohdan Nosyk isa CIHR Bisby Fellow, and also supported by a postdoctoral fellowship from the Michael Smith Foundation for Health Research. Brandon Marshall is supported by postdoctoral fellowships from the CIHR and the International AIDS Society/National Institute on Drug Abuse. All funding bodies had no further role in study design; in the collection, analysis and interpretation of data; in the writing of the report; or in the decision to submit the paper for publication.

\section{References}

Brands B, Blake J, Sproule B, Gourlay D, Busto U. Prescription opioid abuse in patients presenting for methadone maintenance treatment. Drug Alcohol Depend. 2004; 73:199-207. [PubMed: 14725960] 
Bruneau J, Roy E, Arruda N, Zang G, Jutras-Aswad D. The rising prevalence of prescription opioid injection and its association with hepatitis $\mathrm{C}$ incidence among street-drug users. Addiction. 201210.1111/j.1360-0443.2012.03803.x

Caulkins JP. Price and purity analysis for illicit drugs: data and conceptual issues. Drug Alcohol Depend. 2007; 90(Suppl 1):S61-S68. [PubMed: 16971056]

Chou R, Fanciullo GJ, Fine PG. American Pain Society-American Academy of Pain Medicine Opioids Guidelines Panel. Clinical guidelines for the use of chronic opioid therapy in chronic noncancer pain. J Pain. 2009; 10:113-130. [PubMed: 19187889]

College of Physicians and Surgeons of British Columbia. [Accessed: June 1st, 2008] Methadone Maintenance Handbook. Physician License Required. 2005. http://www.bcguidelines.ca/gpac/ guideline_methadone.html

College of Pharmacists of British Columbia. [Accessed Nov 1, 2011] Guidelines for Addressing Pharmacy Robbery in BC. 2011. http://www.bcpharmacists.org/news_events/news/news136.php

Darke S, Kaye S, Topp L. Cocaine use in New South Wales, Australia, 1996-2000: 5 year monitoring of trends in price, purity, availability and use from the illicit drug reporting system. Drug Alcohol Depend. 2002; 67:81-88. [PubMed: 12062781]

Dhalla IA, Mamdani MM, Gomes T, Juurlink DN. Clustering of opioid prescribing and opioid-related mortality among family physicians in Ontario. Can Fam Physician. 2011; 57:e92-96. [PubMed: 21402956]

Fischer B, Rehm J, Brissette S, Brochu S, Bruneau J, El-Guebaly N, Noël L, Tyndall M, Wild C, Mun P, Baliunas D. Illicit opioid use in Canada: comparing social, health, and drug use characteristics of untreated users in five cities (OPICAN study). J Urban Health. 2005; 82:250-266. [PubMed: 15872194]

Fischer B, Jones W, Krahn M, Rehm J. Differences and over-time changes in levels of prescription opioid analgesic dispensing from retail pharmacies in Canada, 2005-2010. Pharmacoepidemiol Drug Saf. 2011; 20:1269-1277. [PubMed: 21755568]

[Accessed Nov 1, 2011] Gang violence in BC linked to Mexico Drug Wars. http://www.ctvbc.ctv.ca/ servlet/an/local/CTVNews/20090304/bc_mexico_drugs_090304/20090304/? hub=BritishColumbiaHome

Gomes T, Mamdani MM, Dhalla IA, Paterson JM, Juurlink DN. Opioid dose and drug-related mortality in patients with nonmalignant pain. Arch Intern Med. 2011; 171:686-691. [PubMed: 21482846]

Gomes T, Juurlink DN, Dhalla IA, Mailis-Gagnon A, Paterson JM, Mamdani MM. Trends in opioid use and dosing among socio-economically disadvantaged patients. Open Med. 2011; 5:e13-e22. [PubMed: 22046214]

Harris KA Jr, Arnsten JH, Joseph H, Hecht J, Marion I, Juliana P, Gourevitch MN. A 5-year evaluation of a methadone medical maintenance program. J Subst Abuse Treat. 2006; 31:433-438. [PubMed: 17084798]

Inciardi JA, Surratt HL, Kurtz SP, Cicero TJ. Mechanisms of prescription drug diversion among druginvolved club- and street-based populations. Pain Med. 2007; 8:171-183. [PubMed: 17305688]

Kreek MJ, Borg L, Ducat E, Ray B. Pharmacotherapy in the treatment of addiction: methadone. J Addict Dis. 2010; 29:200-216. [PubMed: 20407977]

Kurdyak P, Gomes T, Yao Z, Mamdani MM, Hellings C, Fischer B, Rehm J, Bayoumi AM, Juurlink DN. Use of other opioids during methadone therapy: a population-based study. Addiction. 2011; 107:776-780. [PubMed: 22050078]

Marshall BD, Milloy MJ, Wood E, Montaner JS, Kerr T. Reduction in overdose mortality after the opening of North America's first medically supervised safer injecting facility: a retrospective population-based study. Lancet. 2011; 377:1429-1437. [PubMed: 21497898]

Ministry of Public Safety and Solicitor General Police Services Division. [Accessed Nov 1, 2011] Police Resources in British Columbia, 2009. 2011. http://www.pssg.gov.bc.ca/police_services/ publications/statistics/policeresourcesinbc.pdf

National Institute on Drug Abuse. [Accessed January 31st, 2012] Infofacts: Drug-related hospital emergency room visits. http://www.drugabuse.gov/publications/infofacts/drug-related-hospitalemergency-room-visits 
National Centre for Injury Prevention and Control. Centers for Disease Control. Centers for Disease Control and Prevention. [Accessed Nov 1st, 2011] Unintentional drug poisoning in the United States. Jul. 2010 http://www.cdc.gov/HomeandRecreationalSafety/Poisoning/index.html

Popova S, Patra J, Mohapatra S, Fischer B, Rehm J. How many people in Canada use prescription opioids non-medically in general and street drug using populations? Can J Public Health. 2009; 100:104-108. [PubMed: 19839284]

Rosenblum A, Parrino M, Schnoll SH, Fong C, Maxwell C, Cleland CM, Magura S, Haddox JD. Prescription opioid abuse among enrollees into methadone maintenance treatment. Drug Alcohol Depend. 2007; 90:64-71. [PubMed: 17386981]

Roy E, Arruda N, Bourgois P. The growing popularity of prescription opioid injection in downtown Montréal: new challenges for harm reduction. Subst Use Misuse. 2011; 46:1142-1150. [PubMed: 21370963]

Ruel JM, Hickey CP. Diversion of methadone: what are the risks? BC Med J. 1993; 35:420-421.

Salsitz EA, Joseph H, Frank B, Perez J, Richman BL, Salomon N, Kalin MF, Novick DM. Methadone medical maintenance (MMM): treating chronic opioid dependence in private medical practice--a summary report (1983-1998). Mt Sinai J Med. 2000; 67:388-397. [PubMed: 11064489]

Shield KD, Ialomiteanu A, Fischer B, Mann RE, Rehm J. Non-medical use of prescription opioids among Ontario adults: data from the 2008/2009 CAMH Monitor. Can J Public Health. 2011; 102:330-335. [PubMed: 22032096]

Strathdee SA, Palepu A, Cornelisse PG, Yip B, O’Shaughnessy MV, Montaner JS, Schechter MT, Hogg RS. Barriers to use of free antiretroviral therapy in injection drug users. JAMA. 1998; 280:547-549. [PubMed: 9707146]

Strathdee SA, Patrick DM, Currie SL, Cornelisse PG, Rekart ML, Montaner JS, Schechter MT, O'Shaughnessy MV. Needle exchange is not enough: lessons from the Vancouver injecting drug use study. AIDS. 1997; 11:F59-F65. [PubMed: 9223727]

Substance Abuse and Mental Health Service Administration - Center for Behavioural Health Statistics and Quality. [Accessed Jan 31, 2012] http://wwwdasis.samhsa.gov/webt/tedsweb/ tab_year.choose_year_web_table?t_state=US

Tyndall MW, Currie S, Spittal P, Li K, Wood E, O’Shaughnessy MV, Schechter MT. Intensive injection cocaine use as the primary risk factor in the Vancouver HIV-1 epidemic. AIDS. 2003; 17:887-893. [PubMed: 12660536]

Sajan A, Corneil T, Grzybowski S. The street value of prescription drugs. CMAJ. 1998; 159:139-142. [PubMed: 9700324]

US Department of Health and Human Services. Centers for Disease Control and Prevention: Morbidity and Mortality Weekly Report. Vital Signs: Overdoses of Prescription Opioid Pain Relievers United States, 1999-2008. 2011 Nov 1.60 2011. http://responsibleopioidprescribing.org/ index_12_3736347817.pdf.

US Department of Health and Human Services. [Accessed Nov 1, 2011] 2011. http://www.fda.gov/ NewsEvents/Newsroom/PressAnnouncements/ucm207480.htm

Volkow ND, McLellan TA. Curtailing diversion and abuse of opioid analgesics without jeopardizing pain treatment. JAMA 2011. 2011; 305:1346-1347.

Wood E, Tyndall MW, Spittal PM, Li K, Anis AH, Hogg RS, Montaner JS, O’Shaughnessy MV, Schechter MT. Impact of supply-side policies for control of illicit drugs in the face of the AIDS and overdose epidemics: investigation of a massive heroin seizure. CMAJ. 2003; 168:165-169. [PubMed: 12538544]

Wood E, Spittal PM, Small W, Kerr T, Li K, Hogg RS, Tyndall MW, Montaner JS, Schechter MT. Displacement of Canada's largest public illicit drug market in response to a police crackdown. CMAJ. 2004; 170:1551-1556. [PubMed: 15136548]

Wood E, Stoltz JA, Li K, Montaner JS, Kerr T. Changes in Canadian heroin supply coinciding with the Australian heroin shortage. Addiction. 2006; 101:689-695. [PubMed: 16669902]

Wood E, Kerr T. What do you do when you hit rock bottom? Responding to drugs in the city of Vancouver. Int J Drug Policy. 2006; 17:55-60.

Wood E, Stoltz JA, Montaner JS, Kerr T. Evaluating methamphetamine use and risks of injection initiation among street youth: the ARYS study. Harm Reduct J. 2006; 3:18. [PubMed: 16723029] 
Wood E, Kerr T, Marshall BDL, Li K, Zhang R, Hogg RS, Harrigan PR, Montaner JSG. Longitudinal community plasma HIV-1-RNA concentrations and incidence of HIV-1 among injecting drug users: a prospective cohort study. BMJ. 2009; 338:1191-1194. 


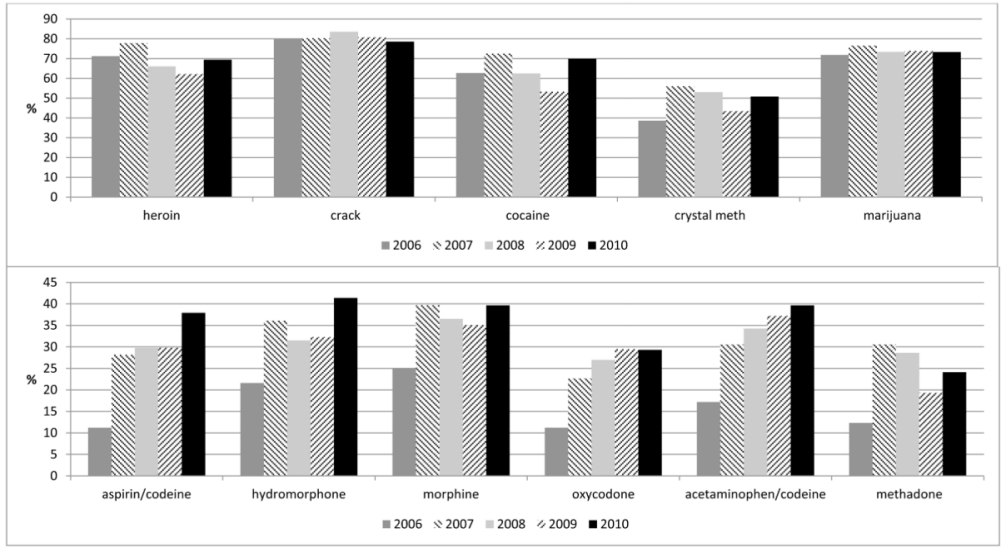

Figure 1. Percentage of ever-users of each drug indicating immediate availability * * Immediately available $=$ available within 10 minutes. Data are derived from baseline interviews of participants entering the cohorts each year. 


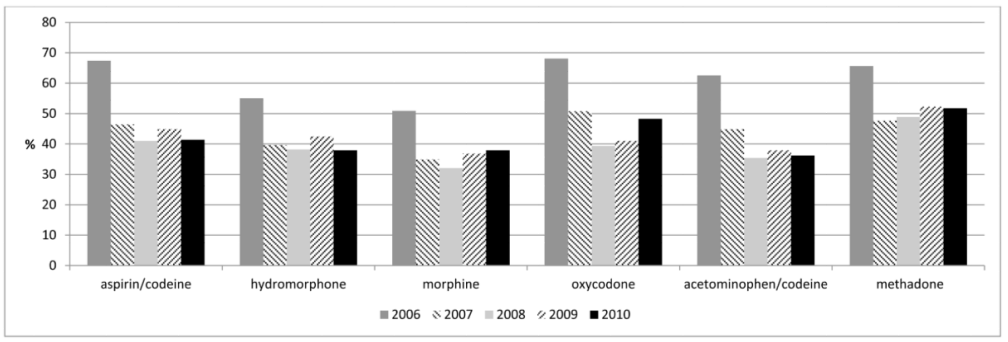

Figure 2.

Percentage of ever-users indicating prescribed opioids were not available 
Table 1

Descriptive characteristics of illicit drug users $(\mathrm{N}=1871)$

\begin{tabular}{lc}
\hline Covariate & N (\%) \\
\hline Female gender & $583(31.2)$ \\
Age: <25 & $695(37.2)$ \\
$25-35$ & $278(14.9)$ \\
$35-45$ & $466(24.9)$ \\
$>$ 45 & $432(23.1)$ \\
Drug dealing ${ }^{*}$ & $703(37.8)$ \\
Sex work ${ }^{*}$ & $155(8.3)$ \\
Unstable housing ${ }^{*}$ & $969(52.0)$ \\
Current DTES residence & $812(43.4)$ \\
DTES residence ${ }^{*}$ & $650(34.7)$ \\
Regular DTES visits ${ }^{*}$ & $448(23.9)$ \\
Purchased drugs in DTES ${ }^{*}$ & $1263(67.5)$ \\
VIDUS Cohort & $623(33.3)$ \\
ACCESS Cohort & $536(28.7)$ \\
ARYS cohort & $712(38.1)$ \\
Year of cohort entry: 2006 & $643(34.4)$ \\
2007 & $329(17.6)$ \\
2008 & $286(15.3)$ \\
2009 & $465(24.9)$ \\
Self-reported ever use in lifetime: Heroin & $1374(73.8)$ \\
Crack cocaine & $1042(55.9)$ \\
\hline & $1653(88.7)$ \\
Prewder cocaine & $1000(53.7)$ \\
\hline & $1235(66.3)$ \\
\hline
\end{tabular}

DTES: Downtown Eastside.

* refers to activities in the past six months. 
